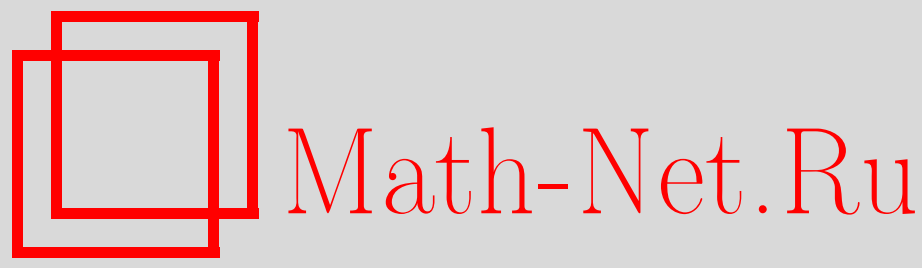

В. И. Алхимов, $d$-Мерная модель канонического ансамбля открытых струн, ТМФ, 2014, том 180, номер 1, 125144

DOI: https://doi.org/10.4213/tmf8633

Использование Общероссийского математического портала Math-Net.Ru подразумевает, что вы прочитали и согласны с пользовательским соглашением http://www . mathnet.ru/rus/agreement

Параметры загрузки:

IP : 52.87 .193 .239

26 апреля 2023 г., 18:13:03

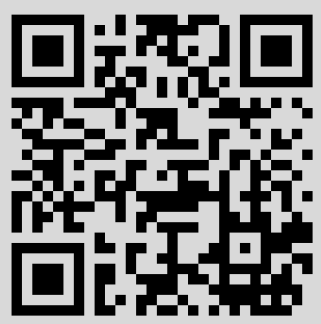




\title{
$d$-МЕРНАЯ МОДЕЛЬ КАНОНИЧЕСКОГО АНСАМБЛЯ ОТКРЫТЫХ СТРУН
}

\begin{abstract}
Предложена $d$-мерная модель канонического ансамбля открытых струн, избегающих самопересечения. Рассмотрена одиночная модель открытой струны в $d$-мерном евклидовом пространстве $\mathbb{R}^{d}, 2 \leqslant d<4$, в котором конфигурация струны описывается ее контурной длиной $L$ и расстоянием $R$ между ее концами. Распределение пространственного размера струны определяется только ее внутренним физическим состоянием и взаимодействием с окружающей средой. Для преобразованной плотности вероятности $W(R, L)$ расстояния $R$ установлено уравнение, аналогичное известному уравнению Дайсона, инвариантное относительно непрерывной группы ренормировочных преобразований, что позволяет использовать метод ренормгруппы для исследования асимптотического поведения указанной плотности, когда $R \rightarrow \infty$ и $L \rightarrow \infty$. Рассмотрена модель ансамбля из $M$ открытых струн со средней по всем струнам контурной длиной $\bar{L}$, и с помощью метода Дарвина-Фаулера получено наиболее вероятное распределение струн по их длинам в пределе $M \rightarrow \infty$. Усреднение плотности вероятности $W(R, L)$ по каноническому ансамблю дает в итоге искомую плотность $\langle W(R, \bar{L})\rangle$.
\end{abstract}

Ключевые слова: $d$-мерная модель, модель открытой струны, основное уравнение, ренормализационная группа, асимптотическое распределение, канонический ансамбль.

DOI: $10.4213 / \operatorname{tmf} 8633$

\section{1. ОДИНОЧНАЯ МОДЕЛЬ ОТКРЫТОЙ СТРУНЫ}

Одной из важных проблем в статистической теории линейных полимеров (макромолекул) является вычисление их средних пространственных размеров. Вследствие очень большого числа степеней свободы у макромолекул последние можно рассматривать как макроскопические системы и для вычисления средних значений величин, характеризующих их размеры, применять статистические методы. Результаты многочисленных экспериментов и теоретических расчетов показывают, что при объяснении макроскопических свойств таких систем мелкомасштабная структура макромолекул с увеличением их длины становится все менее существенной. При

* Московский городской психолого-педагогический университет, Москва, Россия. E-mail: alvaliv@list.ru 
этом ведущую роль в конфигурационной статистике макромолекул играет их цепное строение и связанный с ним эффект "исключенного" объема, согласно которому в одном и том же элементе объема пространства не может находиться одновременно более одного звена (мономера) полимера. Именно эти факторы определяют характерные черты поведения макромолекул и служат основой для их описания с помощью непрерывной модели - модели гибкой открытой струны, избегающей самопересечения.

Пусть исходная модель представляет собой цепь, состоящую из $N$ упорядоченных прямолинейных сегментов, свободное сочленение которых обуславливает ее гибкость. Концы сегментов, являющиеся точками их соединения, будем называть элементами рассматриваемой цепи. Принимая один из концов цепи за нулевой элемент, обозначим через $\mathbf{q}_{i} \in \mathbb{R}^{d}$ радиус-вектор $i$-го элемента, а через $\mathbf{r}_{i}=\mathbf{q}_{i}-\mathbf{q}_{i-1}$ обозначим $i$-й сегмент, соединяющий $i$-й и $(i-1)$-й элементы цепи. Тогда

$$
r_{i j}=\left|\sum_{k=i+1}^{j} \mathbf{r}_{k}\right| \quad \text { и } \quad \bar{r}_{i j}=\sum_{k=i+1}^{j} r_{k}
$$

суть соответственно пространственное расстояние между $i$-м и $j$-м элементами цепи и расстояние по контуру цепи между ними. Мы полагаем, что длины $r_{k}, 1 \leqslant k \leqslant N$, сегментов цепи подчинены одному и тому же распределению, для которого средняя длина каждого сегмента равна $h$, а среднее значение контурной длины $\bar{r}_{i j}$ равно $\tilde{r}_{i j}=|j-i| h$. Отсюда следует, что $\tilde{r}_{0 N}=N h$ есть средняя контурная длина цепи. В дальнейшем при построении непрерывной модели цепи в определении $\tilde{r}_{0 N}$ мы перейдем к пределу, устремив длину $h$ к нулю, а число $N$ к бесконечности таким образом, чтобы было выполнено условие

$$
0<L=\lim _{\substack{h \rightarrow 0, N \rightarrow \infty}} N h<\infty .
$$

В основу статистического описания равновесной конфигурации цепи положим плотность распределения Гиббса в пространстве координат

$$
w\left(\mathbf{q}_{0}, \mathbf{q}_{1}, \ldots, \mathbf{q}_{N}\right)=\frac{1}{Q} e^{-U / T},
$$

которая также называется $(N+1)$-частичной корреляционной функцией. Здесь $Q-$ нормировочный множитель, $U$ - потенциальная энергия рассматриваемой системы, а $T$ - энергетический параметр, характеризующий взаимодействие системы с окружающей средой. Потенциальную энергию $U$ цепи представим в виде суммы двух частей:

$$
U=U_{\mathrm{lin}}+U_{\mathrm{vol}}, \quad U_{\mathrm{lin}}=\sum_{k=1}^{N} u\left(r_{k}\right), \quad U_{\mathrm{vol}}=\sum_{1 \leqslant i<j \leqslant N} \sum_{i j} V_{i j},
$$

где линейная часть $U_{\text {lin }}$ есть сумма внутренних энергий всех сегментов, в то время как объемная часть $U_{\text {vol }}$ обозначает сумму энергий их попарных взаимодействий. Если $u\left(r_{k}\right)$ определяет значение упругой энергии $k$-го сегмента, то величина $V_{i j}$, описывающая пространственное взаимодействие $i$-го и $j$-го сегментов, обуславливает гибкость цепи. Для линейного потенциала $u(r)$ мы примем известное приближение (закон Гука):

$$
u(r)=\frac{\sigma}{2 h} r^{2},
$$


где $\sigma$ - параметр упругости. Величина $V_{i j}$ в этой модели определяется как $V_{i j}=$ $h^{2} V\left(r_{i j}, \tilde{r}_{i j}\right)$, где $V\left(r_{i j}, \tilde{r}_{i j}\right)$ обозначает плотность энергии взаимодействия $i$-го и $j$-го сегментов цепи. Таким образом, энергия взаимодействия $V_{i j}$ двух сегментов зависит здесь как от пространственного расстояния $r_{i j}$, так и от средней контурной длины $\tilde{r}_{i j}$ между ними. Чтобы учесть запрет на самопересечения цепи, определим плотность энергии $V(r, \tilde{r})$ следующим образом:

$$
V(r, \tilde{r})=V_{0} \theta\left(r_{0}-r\right) \theta(\tilde{r}-l)
$$

здесь $V_{0}$ - значение функции $V(r, \tilde{r})$ в области $0 \leqslant r \leqslant r_{0}<l \leqslant \tilde{r}$, где $r_{0}$ и $l$ - положительные параметры рассматриваемой модели, $V_{0}=$ const $>0$, и $\theta(x)$ - функция Хевисайда.

Согласно методу Маркова запишем искомую плотность вероятности расстояния $R$ между концами цепи следующим образом:

$$
W_{N}(\mathbf{R})=\int d \mathbf{q}_{1} \ldots d \mathbf{q}_{N} w\left(\mathbf{q}_{0}, \mathbf{q}_{1}, \ldots, \mathbf{q}_{N}\right) \delta\left(\mathbf{R}+\mathbf{q}_{0}-\mathbf{q}_{N}\right),
$$

где $\delta(\mathbf{q})$ - дельта-функция Дирака. В этом случае один из концов цепи можно считать фиксированным в начале координат, а другой - свободным. Тогда в силу изотропности пространства $\mathbb{R}^{d}$ плотность распределения $W_{N}(\mathbf{R})$ зависит лишь от расстояния $R$ свободного конца цепи от начала координат. Далее в интеграле (4) перейдем к новым переменным $\mathbf{r}_{i}=\mathbf{q}_{i}-\mathbf{q}_{i-1}, i=1, \ldots, N$, и воспользуемся фурьепреобразованием функции $\delta(\mathbf{q})$. В результате получим для $W_{N}(\mathbf{R})$ выражение

$$
W_{N}(\mathbf{R})=\frac{1}{Q_{N}} \int_{\mathbb{R}^{d}} \frac{d \mathbf{p}}{(2 \pi)^{d}} e^{i \mathbf{R} \cdot \mathbf{p}} g_{N}(p),
$$

в котором

$$
Q_{N}=\frac{Q}{Q_{0}^{N}}, \quad Q_{0}=\omega_{d} \int_{0}^{\infty} d r e^{-u(r) / T} r^{2 s+1}, \quad s=\frac{d-2}{2},
$$

$\omega_{d}=2 \pi^{s+1} / \Gamma(s+1)$ - площадь поверхности сферы единичного радиуса в $\mathbb{R}^{d}, \Gamma(x)-$ гамма-функция Эйлера, а функция $g_{N}(\mathbf{p})$ определяется как

$$
g_{N}(\mathbf{p})=\int P_{1 N} \prod_{k=1}^{N} Y_{\mathbf{p}}\left(\mathbf{r}_{k}\right) d \mathbf{r}_{k}
$$

где

$$
\begin{gathered}
P_{1 N}=\exp \left(-\frac{h^{2}}{T} \sum_{1 \leqslant i<j \leqslant N} V\left(r_{i j}, \tilde{r}_{i j}\right)\right), \\
Y_{\mathbf{p}}(\mathbf{r})=\frac{1}{Q_{0}} e^{-i \mathbf{p} \cdot \mathbf{r}-u(r) / T} .
\end{gathered}
$$

Из формул (6)-(8) следует, что

$$
\left|g_{N}(\mathbf{p})\right| \leqslant g_{N}(\mathbf{0})
$$


где величина $g_{N}(\mathbf{0})$ удовлетворяет следующим неравенствам:

$$
\exp \left(-\frac{N^{2} h^{2} V_{0}}{2 T}\right) \leqslant g_{N}(\mathbf{0}) \leqslant 1 .
$$

В отсутствие объемных взаимодействий, т. е. в случае $V_{0}=0$, функция $g_{N}(\mathbf{p})$ в силу равенства (6) преобразуется к виду $g_{N}^{(0)}(\mathbf{p})=g^{N}(\mathbf{p})$, где

$$
g(\mathbf{p})=\int_{\mathbb{R}^{d}} d \mathbf{r} Y_{\mathbf{p}}(\mathbf{r})=\frac{\omega_{d}}{Q_{0}} \int_{0}^{\infty} d r r^{2 s+1} \Lambda_{s}(p r) e^{-u(r) / T},
$$

$\Lambda_{s}(x)=\Gamma(s+1)(2 / x)^{s} J_{s}(x)$ и $J_{s}(x)-$ функция Бесселя. Отсюда получаем $\Lambda_{s}(0)=1$ и $g(0)=1$.

Теперь определим значение указанного выше параметра $l \equiv T / 2 \sigma$ и примем условие $l \gg r_{0}$. Тогда, используя в формуле (11) выражение $(2)$ в виде $u(r) / T=r^{2} / 4 h l$, получим в результате интегрирования следующее равенство: $g(\mathbf{p})=e^{-h l p^{2}}$. Отсюда, пользуясь выражением (5), находим $Q_{N}^{(0)}=1$ и предельное выражение для плотности вероятности:

$$
\lim W_{N}^{(0)}(\mathbf{R}) \equiv W^{(0)}(\mathbf{R}, L)=(4 \pi l L)^{-d / 2} e^{-R^{2} / 4 l L},
$$

где предельный переход осуществляется при условии (1). Найденное с помощью последней формулы значение среднеквадратичного расстояния $\left\langle R^{2}\right\rangle_{L}^{(0)}=2 d l L$ между концами цепи может служить в этом случае дополнением к приведенной выше характеристике величины $l$.

\section{2. ОСНОВНОЕ УРАВНЕНИЕ}

Приступим теперь к поиску асимптотики плотности вероятности

$$
W(\mathbf{R}, L)=\lim W_{N}(\mathbf{R}), \quad \frac{R}{l} \rightarrow \infty, \quad \frac{L}{l} \rightarrow \infty,
$$

при условии (1). С этой целью обратимся к формулам (5), (6) и наряду с определением (7) введем следующие обозначения:

$$
P_{m n}=\prod_{m \leqslant i<j \leqslant n}\left(1+\varepsilon_{i j}\right), \quad \varepsilon_{i j}=e^{-V_{i j} / T}-1 .
$$

Разложим произведение $P_{1 N}$ в ряд по $\varepsilon_{i j}$ :

$$
P_{1 N}=1+\sum_{k \geqslant 1}\left(\sum_{i_{1}<j_{1}} \sum_{i_{2}<j_{2}} \ldots \sum_{i_{k}<j_{k}} \varepsilon_{i_{1} j_{1}} \varepsilon_{i_{2} j_{2}} \ldots \varepsilon_{i_{k} j_{k}}\right),
$$

где индексы в каждом из сомножителей $\varepsilon_{i_{1} j_{1}}, \varepsilon_{i_{2} j_{2}}, \ldots, \varepsilon_{i_{k} j_{k}}$ расположены в порядке возрастания, т. е. $i_{k}<j_{k}$ для $k=1,2, \ldots$, причем первые индексы этих сомножителей в каждом члене указанной суммы образуют неубывающую последовательность, $i_{1} \leqslant i_{2} \leqslant \cdots \leqslant i_{k}$, и $\left(i_{l} j_{l}\right) \neq\left(i_{m} j_{m}\right)$ при $l \neq m$.

Сомножители $\varepsilon_{i_{m} j_{m}}$ и $\varepsilon_{i_{n} j_{n}}$ называются связанными, если контурные участки цепи $\left\{\mathbf{r}_{i_{m}}, \ldots, \mathbf{r}_{j_{m}}\right\}$ и $\left\{\mathbf{r}_{i_{n}}, \ldots, \mathbf{r}_{j_{n}}\right\}$ содержат по крайней мере один общий сегмент, т. е. 
$i_{m} \leqslant i_{n} \leqslant j_{n} \leqslant j_{m}$ или $i_{m} \leqslant i_{n} \leqslant j_{m} \leqslant j_{n}$, и несвязанными в противном случае, при $i_{m} \leqslant j_{m}<i_{n} \leqslant j_{n}$. Любое произведение сомножителей в (15), расположенных в порядке неубывания их первых индексов, назовем комплексом, если в этом произведении каждый сомножитель начиная со второго связан по крайней мере с одним из предшествующих сомножителей. Тогда любой член суммы в (15) является либо комплексом, либо распадается на произведение некоторого числа комплексов. Обозначим через $f_{i j}$ сумму всех комплексов, зависящих от векторов $\mathbf{r}_{i}, \mathbf{r}_{i+1}, \ldots, \mathbf{r}_{j}$, где $i<j$, и назовем ее блоком. Это означает, что

$$
\begin{aligned}
& f_{i j}=\varepsilon_{i j} P_{i j}+\sum_{i<k<l<j} \varepsilon_{i l} \varepsilon_{k j} P_{i k} P_{k l} P_{l j}+ \\
& +\sum_{i<k<l<m<n<j}\left(\varepsilon_{i l} \varepsilon_{k n} \varepsilon_{m j}+\varepsilon_{i n} \varepsilon_{k m} \varepsilon_{l j}+\varepsilon_{i m} \varepsilon_{k j} \varepsilon_{l n}+\varepsilon_{i m} \varepsilon_{k n} \varepsilon_{l j}\right) P_{i k} P_{k l} P_{l m} P_{m n} P_{n j}+\cdots
\end{aligned}
$$

где в правой части под знаком первой суммы фигурирует всего лишь один представитель комплексов второго порядка по $\varepsilon_{i j}$, в то время как под знаком второй суммы находятся четыре неэквивалентных представителя комплексов третьего порядка относительно $\varepsilon_{i j}$. Для полного числа $N_{n}$ неэквивалентных представителей комплексов $n$-го порядка по $\varepsilon_{i j}$ имеет место асимптотическая оценка $N_{n}=O((2 n-1) ! !)$ при $n \rightarrow \infty$. Нетрудно видеть, что произведение $P_{1 N}$ можно разложить по блокам $f_{i j}$,

$$
P_{1 N}=1+\sum_{i<j} f_{i j}+\sum_{i_{1}<j_{1}<i_{2}<j_{2}} f_{i_{1} j_{1}} f_{i_{2} j_{2}}+\cdots,
$$

так, что каждый член суммы является либо блоком, либо произведением некоторого числа блоков. Подставляя это разложение в формулу (6) и выполняя надлежащее интегрирование в каждом ее члене, получим следующее выражение:

$$
g_{N}(\mathbf{p})=\sum_{m=0}^{\infty} \sum_{n=0}^{N} \frac{(N-n+m) !}{(N-n) ! m !} g^{N-n}(\mathbf{p}) F_{n}^{(m)}(\mathbf{p}),
$$

где

$$
\begin{gathered}
F_{n}^{(0)}(\mathbf{p}) \equiv 0, \quad F_{n}^{(m)}(\mathbf{p})=\sum_{n_{1}+\cdots+n_{m}=n} f_{n_{1}}(\mathbf{p}) \ldots f_{n_{m}}(\mathbf{p}), \quad m \geqslant 1 \\
f_{1}(\mathbf{p}) \equiv 0, \quad f_{n}(\mathbf{p})=\int f_{1 n} \prod_{k=1}^{n} Y_{\mathbf{p}}\left(\mathbf{r}_{k}\right) d \mathbf{r}_{k}, \quad n>1
\end{gathered}
$$

и $f_{1 n}$, как указано выше, обозначает блок, который состоит из всех комплексов, зависящих от векторов $\left\{\mathbf{r}_{k}\right\}, k=1, \ldots, n$, а множитель $(N-n+m) ! /(N-n) ! m !$ равен числу способов размещения $m$ частичных отрезков, состоящих последовательно из $n_{1}, \ldots, n_{m}$ звеньев (общее число которых равно $n$ ) и расположенных внутри одного отрезка из $N$ звеньев, при условии, что сохраняется последовательность расположения частичных отрезков.

Воспользуемся формулой Коши

$$
\frac{1}{2 \pi i} \oint_{\gamma} d z z^{n_{1}+\cdots+n_{m}-n-1}= \begin{cases}1, & \text { если } n_{1}+\cdots+n_{m}=n \\ 0, & \text { если } n_{1}+\cdots+n_{m} \neq n\end{cases}
$$


в которой замкнутый контур $\gamma$ охватывает начало координат на плоскости комплексной переменной $z$, и перепишем соотношение (17) в виде

$$
F_{n}^{(m)}(\mathbf{p})=\frac{1}{2 \pi i} \oint_{\gamma} \frac{d z}{z^{n+1}} f^{m}(\mathbf{p}, z),
$$

где

$$
f(\mathbf{p}, z) \equiv \sum_{n=0}^{\infty} z^{n} f_{n}(\mathbf{p})
$$

Далее подставим выражение (20) в (17) и распространим суммирование по $n$ от $-\infty$ до $N$, что не оказывает влияния на конечный результат. Тогда, используя формулу

$$
(1-x-y)^{-1}=\sum_{m=0}^{\infty} \sum_{n=0}^{\infty} \frac{(m+n) !}{m ! n !} x^{m} y^{n}, \quad|x+y|<1,
$$

приведем выражение (17) к виду

$$
g_{N}(\mathbf{p})=\frac{1}{2 \pi i} \oint_{\gamma}[1-z g(\mathbf{p})-f(\mathbf{p}, z)]^{-1} \frac{d z}{z^{N+1}},
$$

где замкнутый контур $\gamma$, охватывающий начало координат $z=0$, выбран так, чтобы выполнялось неравенство

$$
|z g(\mathbf{p})+f(\mathbf{p}, z)|<1 .
$$

Наконец, если определить производящую функцию

$$
g(\mathbf{p}, z) \equiv \sum_{N \geqslant 0} z^{N} g_{N}(\mathbf{p})
$$

то из равенства (22) следует основное уравнение [1]

$$
g^{-1}(\mathbf{p}, z)=g_{0}^{-1}(\mathbf{p}, z)-f(\mathbf{p}, z),
$$

в котором $g_{0}^{-1}(\mathbf{p}, z)=1-z g(\mathbf{p})$. Однако, для того чтобы уравнение (24) было замкнутым, необходимо установить связь между производящими функциями $f(\mathbf{p}, z)$ и $g(\mathbf{p}, z)$. С этой целью подставим выражение для $f_{1 n}$ из общей формулы (16) в равенство $(19)$ и представим $\varepsilon_{m n}$ в следующем виде:

$$
\varepsilon_{m n}=\varepsilon\left(\left|\sum_{k=m+1}^{n} \mathbf{r}_{k}\right|,|m-n| h\right)=\frac{1}{2 \pi i} \oint_{\gamma} \frac{d z}{z^{n-m}} \int \frac{d \mathbf{p}}{(2 \pi)^{d}} \exp \left(-i \mathbf{p} \cdot \sum_{j=m+1}^{n} \mathbf{r}_{j}\right) \tilde{\varepsilon}(\mathbf{p}, z),
$$

где

$$
\tilde{\varepsilon}(\mathbf{p}, z)=\sum_{n=0}^{\infty} z^{n} \int d \mathbf{r} e^{i \mathbf{p} \cdot \mathbf{r}}\left(e^{-\left(h^{2} / T\right) V(r, n h)}-1\right)
$$

в соответствии с определением $\varepsilon_{m n}$ в (14). Если в уравнении (19) выполнить интегрирование по всем $\mathbf{r}_{k}, k=1, \ldots, n$, то получим ряд

$$
\begin{aligned}
f_{n}(\mathbf{p})= & \frac{1}{2 \pi i} \oint_{\gamma} \frac{d z_{1}}{z_{1}^{n+1}} \int \frac{d \mathbf{p}_{1}}{(2 \pi)^{d}} \tilde{\varepsilon}\left(\mathbf{p}_{1}, z_{1}\right) g_{n}\left(\mathbf{p}-\mathbf{p}_{1}\right)+ \\
& +\sum_{1 \leqslant l<m<n} \frac{1}{(2 \pi i)^{2}} \oint_{\gamma} \frac{d z_{1}}{z_{1}^{m+1}} \oint_{\gamma} \frac{d z_{2}}{z_{2}^{n-l+1}} \int \frac{d \mathbf{p}_{1}}{(2 \pi)^{d}} \int \frac{d \mathbf{p}_{2}}{(2 \pi)^{d}} \tilde{\varepsilon}\left(\mathbf{p}_{1}, z_{1}\right) \tilde{\varepsilon}\left(\mathbf{p}_{2}, z_{2}\right) \times \\
& \times g_{l}\left(\mathbf{p}-\mathbf{p}_{1}\right) g_{m-l}\left(\mathbf{p}-\mathbf{p}_{1}-\mathbf{p}_{2}\right) g_{n-m}\left(\mathbf{p}-\mathbf{p}_{2}\right)+\cdots
\end{aligned}
$$


Подставим его в равенство (21) и затем выполним надлежащее суммирование. В результате для $f(\mathbf{p}, z)$ мы получим выражение

$$
\begin{aligned}
f(\mathbf{p}, z)= & \frac{1}{2 \pi i} \oint_{\gamma} \frac{d z_{1}}{z_{1}} \int \frac{d \mathbf{p}_{1}}{(2 \pi)^{d}} \tilde{\varepsilon}\left(\mathbf{p}_{1}, z_{1}\right) g\left(\mathbf{p}-\mathbf{p}_{1}, z / z_{1}\right)+ \\
+ & \frac{1}{(2 \pi i)^{2}} \oint_{\gamma} \frac{d z_{1}}{z_{1}} \oint_{\gamma} \frac{d z_{2}}{z_{2}} \int \frac{d \mathbf{p}_{1}}{(2 \pi)^{d}} \int \frac{d \mathbf{p}_{2}}{(2 \pi)^{d}} \tilde{\varepsilon}\left(\mathbf{p}_{1}, z_{1}\right) \tilde{\varepsilon}\left(\mathbf{p}_{2}, z_{2}\right) \times \\
& \times g\left(\mathbf{p}-\mathbf{p}_{1}, z / z_{1}\right) g\left(\mathbf{p}-\mathbf{p}_{1}-\mathbf{p}_{2}, z / z_{1} z_{2}\right) g\left(\mathbf{p}-\mathbf{p}_{2}, z / z_{2}\right)+\cdots,
\end{aligned}
$$

определяющее искомую связь между функциями $f(\mathbf{p}, z)$ и $g(\mathbf{p}, z)$. Полученный ряд в правой части данного равенства удобно представить в символической форме

$$
f(\mathbf{p}, z)=\sum_{n=1}^{\infty} N_{n} \oint_{\gamma}\left\{\frac{d z^{\prime}}{2 \pi i z^{\prime}}\right\}^{n} \int\left\{\frac{d \mathbf{p}^{\prime}}{(2 \pi)^{d}}\right\}^{n}\left\{\tilde{\varepsilon}\left(\mathbf{p}^{\prime}, z^{\prime}\right)\right\}^{n}\left\{g\left(\mathbf{p}-\mathbf{p}^{\prime}, z / z^{\prime}\right)\right\}^{2 n-1}
$$

с целью продемонстрировать инвариантность уравнения (24) относительно мультипликативных преобразований:

$$
g \rightarrow g^{\prime}=\alpha g, \quad g_{0} \rightarrow g_{0}^{\prime}=\alpha g_{0}, \quad \tilde{\varepsilon} \rightarrow \tilde{\varepsilon}^{\prime}=\alpha^{-2} \tilde{\varepsilon}, \quad f \rightarrow f^{\prime}=\alpha^{-1} f,
$$

где $\alpha$ - отличный от нуля параметр, изменяющийся непрерывно. Эти преобразования образуют непрерывную группу, называемую ренормгруппой (РГ), в связи с чем свойство (26) является исходной точкой для применения метода РГ в исследуемой задаче.

\section{3. ПЕРЕХОД К НЕПРЕРЫВНОЙ МОДЕЛИ}

Чтобы осуществить переход от дискретной модели цепи к непрерывной, называемой в дальнейшем моделью открытой струны, введем новую переменную $E$ посредством равенства

$$
z=z(E) \equiv e^{-E h}, \quad \operatorname{Re} E>0,
$$

и, применяя свойство РГ-инвариантности (26), запишем уравнение (24) в форме

$$
[h g(\mathbf{p}, z(E))]^{-1}=h^{-1}\left(1-e^{-h\left(E+l p^{2}\right)}\right)-h^{-1} f(\mathbf{p}, z(E)) .
$$

Тогда в силу неравенства

$$
|g(\mathbf{p}, z(E))|<\frac{1}{1-e^{-h \operatorname{Re} E}},
$$

вытекающего из соотношений (9), (10), (24) и (27), функция $(1-z) g(\mathbf{p}, z)$, аналитическая в области $|z|<1$, является ограниченной внутри этой области, и поэтому существует предел [2]

$$
\lim _{h \rightarrow 0} h g(\mathbf{p}, z(E)) \equiv \varphi(\mathbf{p}, E) .
$$

Из формул (29) и (30) следует неравенство

$$
|\varphi(\mathbf{p}, E)| \leqslant \frac{1}{\operatorname{Re} E} .
$$


Далее воспользуемся равенствами $z^{-1}(E) d z(E)=-h d E$ и

$$
h^{-1} \tilde{\varepsilon}(\mathbf{p}, z(E))=\sum_{n=0}^{\infty} h e^{-n h E} \int_{\mathbb{R}^{d}} d \mathbf{r} e^{i \mathbf{p} \cdot \mathbf{r}}\left(e^{-\left(h^{2} / T\right) V(r, n h)}-1\right) h^{-2},
$$

вытекающими из формул (27) и (25) соответственно, и затем перейдем к пределу в уравнении (32) при $h \rightarrow 0$. Тогда, принимая во внимание соотношения (26), (28), (30), (32) и определение

$$
\lim _{h \rightarrow 0} h^{-1} \tilde{\varepsilon}(\mathbf{p}, z(E)) \equiv v(\mathbf{p}, E)=-\frac{1}{T} \int_{\mathbb{R}^{d}} d \mathbf{r} \int_{0}^{\infty} d \tilde{r} e^{i \mathbf{p} \cdot \mathbf{r}-E \tilde{r}} V(r, \tilde{r}),
$$

мы получим для функции $\varphi(\mathbf{p}, E)$ уравнение

$$
\varphi^{-1}(\mathbf{p}, E)=\varphi_{0}^{-1}(\mathbf{p}, E)-F(\mathbf{p}, E ; v ; \varphi),
$$

где $\varphi_{0}^{-1}(\mathbf{p}, E)=E+l p^{2}$, а функция $F(\mathbf{p}, E ; v ; \varphi)$ определяется с помощью ряда:

$$
\begin{aligned}
& F(\mathbf{p}, E ; v ; \varphi)=\int \frac{d \mathbf{p}_{1}}{(2 \pi)^{d}} \int_{C-i \infty}^{C+i \infty} \frac{d E_{1}}{2 \pi i} v\left(\mathbf{p}_{1}, E_{1}\right) \varphi\left(\mathbf{p}-\mathbf{p}_{1}, E-E_{1}\right)+ \\
& \quad+\int \frac{d \mathbf{p}_{1}}{(2 \pi)^{d}} \int \frac{d \mathbf{p}_{2}}{(2 \pi)^{d}} \int_{C-i \infty}^{C+i \infty} \frac{d E_{1}}{2 \pi i} \int_{C-i \infty}^{C+i \infty} \frac{d E_{2}}{2 \pi i} v\left(\mathbf{p}_{1}, E_{1}\right) v\left(\mathbf{p}_{2}, E_{2}\right) \times \\
& \quad \times \varphi\left(\mathbf{p}-\mathbf{p}_{1}, E-E_{1}\right) \varphi\left(\mathbf{p}-\mathbf{p}_{1}-\mathbf{p}_{2}, E-E_{1}-E_{2}\right) \varphi\left(\mathbf{p}-\mathbf{p}_{2}, E-E_{2}\right)+\cdots
\end{aligned}
$$

Согласно равенству (33) фигурирующая в $(35)$ функция $v(\mathbf{p}, E)$ есть $(d+1)$-мерная спектральная плотность функции $-V(r, \tilde{r}) / T$. Подставляя выражение $(3)$ в равенство (33), находим

$$
v(\mathbf{p}, E)=-v_{0} \Lambda_{s+1}\left(p r_{0}\right) E^{-1} e^{-E l},
$$

где $v_{0}=\vartheta V_{0} / T$, а $\vartheta=\left(\pi r_{0}^{2}\right)^{s+1} / \Gamma(s+2)$ есть $d$-мерный объем шара радиуса $r_{0}$.

Подставим теперь выражение (36) во все члены ряда (35) и выполним в них соответствующие интегрирования по переменным $E_{k}, k=1,2, \ldots$. С этой целью в комплексной плоскости каждой из переменных $E_{k}$ проведем разрез вдоль положительной части вещественной оси и сместим вертикальный контур интегрирования $(C-i \infty, C+i \infty)$ по переменной $E_{k}$ бесконечно далеко в правую полуплоскость, обходя при этом особые точки подынтегральных функций. Если теперь выбрать значение параметра $l$ достаточно большим, то из соотношений (31) и (36) следует, что основной вклад в интегралы вносят особые точки $E_{k}=0, k=1,2, \ldots$. Тогда, удерживая вклад в значения интегралов в (35) от указанных особых точек, мы приходим к равенству

$$
\begin{aligned}
F(\mathbf{p}, E ; v ; \varphi)=- & v_{0} \int \frac{d \mathbf{p}_{1}}{(2 \pi)^{d}} \Lambda_{s+1}\left(r_{0} p_{1}\right) \varphi\left(\mathbf{p}-\mathbf{p}_{1}, E\right)+ \\
& \left.+v_{0}^{2} \int \frac{d \mathbf{p}_{1}}{(2 \pi)^{d}} \int \frac{d \mathbf{p}_{2}}{(2 \pi)^{d}} \Lambda_{s+1}\left(r_{0} p_{1}\right) \Lambda_{s+1}\left(r_{0} p_{2}\right)\right) \times \\
& \quad \times \varphi\left(\mathbf{p}-\mathbf{p}_{1}, E\right) \varphi\left(\mathbf{p}-\mathbf{p}_{1}-\mathbf{p}_{2}, E\right) \varphi\left(\mathbf{p}-\mathbf{p}_{2}, E\right)-\cdots
\end{aligned}
$$


Последнее выражение представим в символической форме

$$
F(\mathbf{p}, E ; v ; \varphi)=\sum_{n=1}^{\infty} N_{n}\left(-v_{0}\right)^{n} \int\left\{\frac{d \tilde{\mathbf{p}}}{(2 \pi)^{d}}\right\}^{n}\left\{\Lambda_{s+1}\left(r_{0} \tilde{p}\right)\right\}^{n}\{\varphi(\mathbf{p}-\tilde{\mathbf{p}}, E)\}^{2 n-1},
$$

из которой легко видеть РГ-инвариантность уравнения (34):

$$
\varphi \rightarrow \varphi^{\prime}=\alpha \varphi, \quad \varphi_{0} \rightarrow \varphi_{0}^{\prime}=\alpha \varphi_{0}, \quad v_{0} \rightarrow v_{0}^{\prime}=\alpha^{-2} v_{0}, \quad F \rightarrow F^{\prime}=\alpha^{-1} F .
$$

ПреДЛОЖЕНИЕ 1. Величина $F(0, E ; v ; \varphi)$ удовлетворяет неравенству

$$
\operatorname{Re} E \leqslant|E-F(0, E ; v ; \varphi)| \leqslant L^{-1} \exp \left(\frac{L^{2} V_{0}}{6 T}+\frac{L}{2} \operatorname{Re} E\right) .
$$

ДокАЗАТЕЛЬСтво. Согласно неравенствам (10) и определению (27) имеем

$$
\sum_{n=0}^{N} \exp \left(-\frac{n^{2} h^{2} V_{0}}{2 T}-n h \operatorname{Re} E\right) \leqslant \sum_{n=0}^{N}|z(E)|^{n} g_{n}(0) \leqslant \sum_{n=0}^{N}|z(E)|^{n} .
$$

Тогда, преобразуя левую часть в (40) с помощью известного неравенства

$$
\left(\prod_{n=1}^{N} t_{n}\right)^{1 / N} \leqslant \frac{1}{N} \sum_{n=1}^{N} t_{n}, \quad t_{n}>0
$$

мы без труда замечаем, что она не меньше величины

$$
N \exp \left(-\frac{N^{2}-1}{6} \frac{h^{2} V_{0}}{T}-\frac{N+1}{2} h \operatorname{Re} E\right)
$$

Учитывая эту оценку, умножим все части неравенств в (40) на $h$ и в полученной системе неравенств перейдем к пределу, устремляя длину $h$ к нулю, а число $N$ к бесконечности так, чтобы выполнялось условие (1). В результате мы приходим к соотношениям

$$
L \exp \left(-\frac{L^{2} V_{0}}{6 T}-\frac{L}{2} \operatorname{Re} E\right) \leqslant|\varphi(0, E)| \leqslant \frac{1}{\operatorname{Re} E},
$$

которые с учетом уравнения (34) доказывают неравенства (39).

Из доказанного утверждения следует, что ряд в правой части равенства (37) сходится при $p=0$, и это обуславливает также сходимость интегралов в членах этого ряда. Тогда, принимая во внимание асимптотические формулы

$$
\begin{aligned}
& \Lambda_{s+1}\left(r_{0} p\right) \rightarrow 1, \quad p \rightarrow 0, \\
& \Lambda_{s+1}\left(r_{0} p\right)=O\left(p^{-s-3 / 2}\right), \quad p \rightarrow \infty,
\end{aligned}
$$

можно описать асимптотическое поведение функции $\varphi(\mathbf{p}, 0)$ на концах интервала $0<p<\infty$. Действительно, пусть $E_{0}-$ наиболее удаленная вправо особая точка функции $\varphi(0, E)$, определяемая согласно уравнению (34) равенством

$$
\varphi^{-1}\left(0, E_{0}\right) \equiv 0
$$


или, что эквивалентно, уравнением $E_{0}-F\left(0, E_{0} ; v ; \varphi\right) \equiv 0$. Но из неравенств $(41)$ и уравнения (42) вытекает, что $E_{0}=0$, следовательно, $F(0,0 ; v ; \varphi)=0$. С другой стороны, уравнение (34) при $E=0$ принимает вид

$$
\varphi^{-1}(\mathbf{p}, 0)=l p^{2}-F(\mathbf{p}, 0 ; v ; \varphi) .
$$

Отсюда можно заключить, что запрет на самопересечения струны приведет к распределению ее размера, существенно отличному от нормального, только в том случае, когда поведение функции $\varphi^{-1}(\mathbf{p}, 0)$ на интервале $0<p<\infty$ будет определяться главным образом зависимостью функционала $F(\mathbf{p}, 0 ; v ; \varphi)$ от $p$. Последнее условие означает, что при $p \rightarrow 0$

$$
F(\mathbf{p}, 0 ; v ; \varphi)=O\left(p^{2(1-\mu)}\right) \quad \text { или } \quad \varphi(\mathbf{p}, 0)=O\left(p^{2(\mu-1)}\right),
$$

где $0<\mu<1$, а при $p \rightarrow \infty$

$$
p^{-2} F(\mathbf{p}, 0 ; v ; \varphi) \rightarrow \infty \text { или } \quad p^{2} \varphi(\mathbf{p}, 0) \rightarrow 0 .
$$

Но из формул (37) и (38) следует, что при $E=0$ и $p=0$ всякий переход от одного члена ряда к следующему сопровождается добавлением интеграла вида

$$
I\left(\mathbf{p}_{1}\right)=-v_{0} \int \frac{d \mathbf{p}_{2}}{(2 \pi)^{d}} \Lambda_{s+1}\left(r_{0} p_{2}\right) \varphi\left(\mathbf{p}_{2}, 0\right) \varphi\left(\mathbf{p}_{2}+\mathbf{p}_{1}, 0\right) .
$$

Вычисление последнего интеграла по малому объему шара радиуса $p_{2}=p_{0}$ с центром в точке $\mathbf{p}_{2}=\mathbf{0}$ при фиксированном значении $\mathbf{p}_{1}=\mathbf{0}$ дает величину порядка $O\left(p_{0}^{4 \mu+2 s-2}\right)$ при $p_{0} \rightarrow 0$. Отсюда вытекает следующее условие сходимости указанного интеграла: $1-s<2 \mu$. Очевидно, что последнее условие содержательно лишь при $s \leqslant 1$. Таким образом, запрет на самопересечения открытой струны приводит в асимптотическом случае к распределению расстояния между ее концами, существенно отличающемуся от нормального распределения, при $d \leqslant 4$ (для $s \leqslant 1$ ).

\section{4. УРАВНЕНИЯ РГ}

Воспользуемся теперь формулой обращения для производящей функции (23) с целью выразить функцию $g_{N}(\mathbf{p})$ через $g(\mathbf{p}, z)$ и затем перейдем к пределу в $(13)$, принимая во внимание равенства $(27)$ и (30). В результате получим

$$
\begin{aligned}
W(\mathbf{R}, L) & =\int_{C-i \infty}^{C+i \infty} \frac{d E}{2 \pi i} e^{L E} \psi(\mathbf{R}, E), \\
\psi(\mathbf{R}, E) & =\int_{\mathbb{R}^{d}} \frac{d \mathbf{p}}{(2 \pi)^{d}} e^{-i \mathbf{R} \cdot \mathbf{p}} \varphi(\mathbf{p}, E) .
\end{aligned}
$$

Более реалистичной задачей является поиск асимптотики функции $W(\mathbf{R}, L)$ при $R / l \rightarrow \infty$ и $L / l \rightarrow \infty$. В этом случае из формул (43), (44) следует, что для ее решения необходимо знать поведение функции $\varphi(\mathbf{p}, E)$ при малых значениях $p$ и $|E|$. Обозначим через $p_{ \pm} \equiv \pm i \eta$ (здесь $\operatorname{Re} \eta>0$ ) ближайшие к началу координат $p=0$ корни уравнения $\varphi^{-1}(\mathbf{p}, E)=0$. Тогда, полагая $p=p_{+}$в уравнении $(34)$, получим тождество

$$
\varphi^{-1}(i \eta, E) \equiv 0
$$


которое вместе с тождеством (42) определяет связь между переменными $E$ и $\eta^{2}$ :

$$
E-E_{0} \equiv l \eta^{2}+F(i \eta, E ; v ; \varphi)-F\left(0, E_{0} ; v ; \varphi\right)
$$

или, в более компактной форме,

$$
E \equiv l \eta^{2}+F(i \eta, E ; v ; \varphi) .
$$

Тем не менее в случае приближенного решения основного уравнения форма связи между $E$ и $\eta^{2}$ вида (46) оказывается удобнее чем (47).

Наконец, с помощью тождества (45) преобразуем уравнение (34) к виду

$$
\varphi^{-1}=l \chi^{2}+F(i \eta, E ; v ; \varphi)-F(p, E ; v ; \varphi),
$$

где $\chi^{2} \equiv p^{2}+\eta^{2}$. Система уравнений (46) (или (47)) и (48) составляет основу для получения указанной выше асимптотики как функции $W(\mathbf{R}, L)$, так и плотности вероятности $\mathcal{W}(\mathbf{R}, L)$ расстояния $R$ между концами струны, контурная длина которой равна $L$. Однако, чтобы вывести выражение для $\mathcal{W}(\mathbf{R}, L)$, необходимо выполнить нормировку функции $W(\mathbf{R}, L)$. В результате с помощью равенств (43) и (44) мы получим для плотности вероятности $\mathcal{W}(\mathbf{R}, L)$ следующую формулу:

$$
\mathcal{W}(\mathbf{R}, L)=\left[\int_{C-i \infty}^{C+i \infty} d E e^{L\left(E-E_{0}\right)} \varphi(0, E)\right]^{-1} \int_{C-i \infty}^{C+i \infty} d E e^{L\left(E-E_{0}\right)} \psi(\mathbf{R}, E) .
$$

Поиск указанной асимптотики плотности $\mathcal{W}(\mathbf{R}, L)$ сводится по существу к вычислению показателя степени $\mu, 0<\mu<1$, в асимптотическом поведении функции $\varphi$,

$$
\varphi=O\left(\chi^{2(\mu-1)}\right), \quad \chi \rightarrow 0,
$$

и установлению асимптотической зависимости между переменными $\eta$ и $E-E_{0}$,

$$
E-E_{0}=O\left(\eta^{2 \nu}\right), \quad \eta \rightarrow 0
$$

где $0<\nu<1$. Для реализации этого подхода воспользуемся РГ-инвариантностью уравнения (48). С этой целью разделим обе части уравнения (48) на $l \chi^{2}$ и представим искомую функцию $\varphi$ как

$$
\varphi=\frac{X}{l \chi^{2}}
$$

где новая неизвестная функция $X$ удовлетворяет уравнению

$$
X^{-1}=1+\frac{\Psi\left(\chi^{2}, \eta^{2} ; v ; \varphi\right)}{l \chi^{2}}
$$

в котором

$$
\Psi\left(\chi^{2}, \eta^{2} ; v ; \varphi\right)=F(i \eta, E ; v ; \varphi)-F(p, E ; v ; \varphi) .
$$

Предположим теперь, что при некотором не равном нулю значении $\chi^{2}=\lambda$ выполняется равенство $\Psi\left(\lambda, \eta^{2} ; v ; \varphi\right)=0$, где $\lambda=\lambda\left(\eta^{2} ; v_{0}\right)$ - так называемая точка нормировки. Тогда уравнение (52) можно переписать в эквивалентной форме

$$
X^{-1}=1+\left(\frac{\lambda}{\chi^{2}}\right) \widetilde{\Psi}\left(\frac{\chi^{2}}{\lambda}, \frac{\eta^{2}}{\lambda} ; \frac{v_{0}}{l^{2} \lambda^{1-s}} ; \frac{\lambda}{\chi^{2}} X\right) .
$$


Отсюда следует, что безразмерная величина $X$ может быть представлена в окрестности точек $p=0$ и $E=0$ как функция $X=X(x, y ; b)$ от безразмерных переменных

$$
x=\frac{\chi^{2}}{\lambda}, \quad y=\frac{\eta^{2}}{\lambda}, \quad b=\frac{v_{0}}{l^{2} \lambda^{1-s}},
$$

где число $b$ представляет собой безразмерную комбинацию величин $\lambda, l$ и $v_{0}$. Действительно, если в каждом интеграле формулы (38) перейти к безразмерным переменным $\tilde{p}^{2} / \lambda$ и $\left(\lambda / \chi^{2}\right) X$, что удобно записать в символической форме

$$
\tilde{p}^{d}=\lambda^{d / 2}\left\{\frac{\tilde{p}^{2}}{\lambda}\right\}^{d / 2}, \quad \varphi^{2}=\frac{1}{(\lambda l)^{2}}\left\{\frac{\lambda}{\chi^{2}} X\right\}^{2},
$$

то в результате такой замены переменных перед интегралом появится множитель $-b$. При этом в точке $x=1$ выполняется условие нормировки $X(1, y ; b)=1$. Отсюда, учитывая РГ-свойство уравнения (52), нетрудно видеть, что умножение функции $X=X(x, y ; b)$ на отличное от нуля число $a^{-1}$ приводит к изменению ее точки нормировки, $\chi^{2}=\lambda^{\prime}\left(\eta^{2}, v_{0}^{\prime}\right)$, и параметра $b, b^{\prime}=a^{-2} \tau^{s-1} b$, где $v_{0}^{\prime}=a^{-2} v_{0}, \tau=\lambda^{\prime} / \lambda$, т. е. $a X(x, y ; b)=X\left(x / \tau, y / \tau ; b^{\prime}\right)$. Полагая $x=\tau$ в последнем равенстве и учитывая условие нормировки $X\left(1, y / \tau ; b^{\prime}\right)=1$, мы получим, что $a^{-1}=X(\tau, y ; b)$, а последнее равенство преобразуется к виду

$$
X(x, y ; b)=X(\tau, y ; b) X\left(\frac{x}{\tau}, \frac{y}{\tau} ; b \tau^{s-1} X^{2}(\tau, y ; b)\right) .
$$

Далее возведем в квадрат обе части данного уравнения и полученный результат умножим на $b x^{s-1}$. Тогда мы приходим к заключению, что величина

$$
B(x, y ; b)=b x^{s-1} X^{2}(x, y ; b),
$$

которую мы назовем инвариантным параметром РГ, удовлетворяет уравнению

$$
B(x, y ; b)=B\left(\frac{x}{\tau}, \frac{y}{\tau} ; B(\tau, y ; b)\right)
$$

с условием нормировки

$$
B(1, y ; b)=b .
$$

Уравнение (55) замкнуто и может быть решено в общем виде [3], [4]. Однако для практических целей более удобно иметь дело с дифференциальным уравнением Ли, соответствующим непрерывной РГ:

$$
x \frac{\partial B(x, y ; b)}{\partial x}=\beta\left(\frac{y}{x} ; B(x, y ; b)\right),
$$

где

$$
\beta(y ; b)=\left.\frac{\partial B(x, y ; b)}{\partial x}\right|_{x=1},
$$

а равенство (56) играет роль граничного условия для уравнения (57). В частном случае, когда $y \rightarrow 0$, уравнение для функции

$$
B(x ; b)=\lim _{y \rightarrow 0} B(x, y ; b)
$$


приводится к уравнению

$$
x \frac{\partial B(x ; b)}{\partial x}=\beta(B(x ; b))
$$

или к уравнению в интегральной форме [5]

$$
\int_{b}^{B(x ; b)} \frac{d a}{\beta(a)}=\ln x, \quad \beta(b)=\left.\frac{\partial B(x ; b)}{\partial x}\right|_{x=1} .
$$

При этом соответствующее граничное условие преобразуется к виду $B(1 ; b)=b$.

Таким образом, инвариантный параметр $B$ является эффективным параметром, характеризующим объемный эффект в малой окрестности точек $p=0$ и $\eta=0$. Его определение позволит в свою очередь описать асимптотическое поведение функции $\varphi$ в соответствии с формулой (50). Обычно для этой цели используется теория возмущений, с помощью которой удается получить информацию о поведении функции $\beta(b)$ лишь в малой окрестности точки $b=0$, где $\beta(b)=0$. В самом деле, если в этой окрестности значение $\beta(b)$ положительно, то $B(x ; b) \rightarrow 0$ при $x \rightarrow 0$, т. е. точка $b=0$ является устойчивым нулем. Если же $\beta(b)$ принимает отрицательные значения вблизи нуля, то величина $B(x ; b)$ возрастает при $x \rightarrow 0$, и теория возмущений становится неприменимой. Используя равенства (54) и (52), мы заключаем, что $B(x ; b) \rightarrow 0$ при $x \rightarrow 0$, если $X(x, b)=o\left(x^{(1-s) / 2}\right)$ при $x \rightarrow 0$. Отсюда следует, что если $X(x, b)=O\left(x^{\mu}\right)$ при $x \rightarrow 0$, то в силу соотношений (50) и (51) неравенство

$$
1-s<2 \mu
$$

является необходимым условием для определения функции $\beta(b)$ в окрестности $b=0$.

Выше было показано, что условие (58) также необходимо для сходимости интегралов во всех членах ряда, представляющего функцию $F(\mathbf{p}, E ; v ; \varphi)$ в окрестности точек $\mathbf{p}=0$ и $E=0$. Но, поскольку значение $\mu$ не зависит от безразмерной переменной $y$, поведение функции $X(x, y ; b)$ при $x \rightarrow 0$ тоже должно описываться подобным образом: $X(x, y ; b)=O\left(x^{\mu}\right)$. Тогда согласно асимптотике (50) для искомой функции $\varphi$ мы имеем $\varphi=O\left(x^{\mu-1}\right)$ при $x \rightarrow 0$. Таким образом, задача сводится к оценке функции $F(\mathbf{p}, 0 ; v ; \varphi)$ в малой окрестности точки $p=0$.

\section{5. АСИМПТОТИКА ПЛОТНОСТИ ВЕРОЯТНОСТИ}

Выше было отмечено, что в случае $d<4$ условие отсутствия самопересечений струны приводит к тому, что величина $\Psi\left(\chi^{2}, \eta^{2} ; v ; \varphi\right)$, определенная в $(53)$, должна вносить основной вклад в правую часть уравнения (48). Поскольку в окрестностях точек $p=0$ и $\eta=0$ переменные $p$ и $\eta$ присутствуют в (48) лишь в виде комбинации $p^{2}+\eta^{2} \equiv \chi^{2}$, естественно предположить, что функция $\Psi\left(\chi^{2}, \eta^{2} ; v ; \varphi\right)$ в окрестности точек $p_{ \pm} \equiv \pm i \eta$, т. е. при $\chi=0$, ведет себя, как $O\left(\chi^{2(1-\mu)}\right)$, где $0<\mu<1$, и растет быстрее чем $\chi^{2}$, когда $\chi^{2} \rightarrow \infty$. Подобное поведение функции $\Psi\left(\chi^{2}, \eta^{2} ; v ; \varphi\right)$ можно описать следующим образом:

$$
\Psi\left(\chi^{2}, \eta^{2} ; v ; \varphi\right) \sim a \chi^{1-\mu} K_{1-\mu}^{-1}\left(a^{\prime} \chi\right)
$$


где $K_{\kappa}(z)$ - функция Макдональда, $a$ и $a^{\prime}$ - некоторые положительные параметры задачи. Функция $K_{\kappa}(z), 0<\kappa<1$, используется здесь благодаря своим асимптотическим свойствам

$$
\begin{array}{rlrl}
\frac{2^{1-\kappa}}{\Gamma(\kappa)} z^{\kappa} K_{\kappa}(z) & =1-\frac{\Gamma(1-\kappa)}{\Gamma(1+\kappa)}\left(\frac{z}{2}\right)^{2 \kappa}+\frac{1}{1-\kappa}\left(\frac{z}{2}\right)^{2}+O\left(z^{2(1+\kappa)}\right), & & z \rightarrow 0 \\
K_{\kappa}(z) & =\sqrt{\frac{\pi}{2 z}} e^{-z}\left(1+O\left(z^{-1}\right)\right), & |z| \rightarrow \infty
\end{array}
$$

адекватно передающим предполагаемое поведение функции $\Psi$. Кроме того, функция $K_{\kappa}(z)=K_{-\kappa}(z)$ при каждом фиксированном значении $z \neq 0$ является целой функцией от индекса $\kappa$, функционально связанного с размерностью $d$ пространства $\mathbb{R}^{d}$. Поэтому исходя из уравнения (48), соотношения (50) и описанного выше поведения функции $\Psi$ мы примем в качестве исходного приближения для искомой функции $\varphi(\mathbf{p}, E)$ выражение

$$
\tilde{\varphi}(p, \eta)=A \chi^{2(\mu-1)} \bar{K}_{1-\mu}(l \chi)
$$

в котором $\bar{K}_{\kappa}(z)=z^{\kappa} K_{\kappa}(z)$, а значения параметров $A$ и $\mu=\mu(d)$, где $0<\mu<1$ для $2 \leqslant d<4(0 \leqslant s<1)$, еще следует найти. Тогда согласно формулам (44) и (59) соответствующее приближение для функции $\psi(\mathbf{R}, E)$ имеет вид

$$
\widetilde{\psi}(\mathbf{R}, \eta)=(2 \pi)^{-d / 2} A \varrho^{-2 \nu} \bar{K}_{\nu}(\eta \varrho),
$$

где $\varrho^{2}=l^{2}+R^{2}, \nu \equiv \mu+s$, при этом $(s+1) / 2<\nu<1$.

Чтобы найти значение параметра $\mu$, воспользуемся следующим способом. В соответствии с формулой (49), а также с принятым приближением (59) и, как следствие, приближением (60) запишем для плотности вероятности $\mathcal{W}(\mathbf{R}, L)$ приближение

$$
\widetilde{\mathcal{W}}(\mathbf{R}, L)=\left[\int_{C-i \infty}^{C+i \infty} d E e^{L\left(E-E_{0}\right)} \tilde{\varphi}(0, \eta)\right]^{-1} \int_{C-i \infty}^{C+i \infty} d E e^{L\left(E-E_{0}\right)} \tilde{\psi}(\mathbf{R}, \eta)
$$

для которого получим асимптотическую оценку, когда $L / l \rightarrow \infty$, но значение $R / l$ при этом остается фиксированным.

Поскольку плотность $\widetilde{\mathcal{W}}(\mathbf{R}, L)$ положительна и, следовательно,

$$
\operatorname{Im} \widetilde{\mathcal{W}}(\mathbf{R}, L)=0
$$

условие (62) имеет место и для ее асимптотики. Для реализации предложенного подхода обратимся к уравнению (46) и выполним оценку функции $F(p, E ; v ; \varphi)$ в окрестностях точек $p=0$ и $E=E_{0}$ (при $\eta=0$ ), используя исходное приближение $\widetilde{\varphi}(p, \eta)$ в равенстве $(37)$. В результате получим

$$
F(p, E ; v ; \tilde{\varphi})=\alpha(p)+k(p) \eta^{2 \nu}+O\left(l^{2} \eta^{2}\right)
$$


где

$$
\begin{gathered}
\alpha(p)=-A_{0} l^{-2 \nu} \tilde{v}(p)+A_{1} l^{2(1+s-3 \nu)} \tilde{v}^{2}(p) \bar{K}_{3 \nu-s-1}(l p), \\
k(p)=\frac{A_{0} \Gamma(1-\nu)}{4^{\nu} \Gamma(1+\nu)} \tilde{v}(p)-A_{2} l^{2(1+s-2 \nu)} \tilde{v}^{2}(p) \bar{K}_{2 \nu-s-1}(l p), \\
\tilde{v}(p)=v_{0} \Lambda_{s+1}(p), \\
A_{0}=\frac{A \Gamma(\nu)}{2^{1-\nu}(2 \pi)^{d / 2}}, \quad A_{1}=\frac{A^{3} \Gamma^{3}(\nu)}{4(2 \pi)^{d} \Gamma(3 \nu)}, \quad A_{2}=\frac{3 A^{3} \Gamma^{3}(\nu) \Gamma(1-\nu)}{2^{2+\nu}(2 \pi)^{d} \Gamma(2 \nu) \Gamma(1+\nu)} .
\end{gathered}
$$

Подставляя выражение (63) в равенство (46), находим

$$
E-E_{0}=\alpha(i \eta)-\alpha(0)+(k(i \eta)-k(0)) \eta^{2 \nu}+O\left(l^{2} \eta^{2}\right)
$$

откуда следует, что

$$
E-E_{0}=k_{0} \eta^{2 \nu}+O\left(\eta^{2(3 \nu-s-1)}\right), \quad \eta \rightarrow 0,
$$

где $k_{0} \equiv k(0)>0$.

Теперь в интегралах формулы (61) введем новую переменную интегрирования $\xi$ посредством равенства $E-E_{0}=k_{0} \xi$. Тогда из соотношения (64) имеем

$$
\xi=\eta^{2 \nu}+O\left(\eta^{2(3 \nu-s-1)}\right), \quad \eta \rightarrow 0
$$

Далее проведем разрез в комплексной плоскости переменной $\xi$ вдоль отрицательной части вещественной оси и в обоих интегралах формулы (61) сдвинем контур интегрирования $(C-i \infty, C+i \infty)$ по $\xi$ бесконечно далеко в левую полуплоскость, обходя при этом особые точки подынтегральных функций, среди которых точка $\xi=0$ имеет наибольшую вещественную часть. Поэтому максимальный вклад в $\widetilde{\mathcal{W}}(\mathbf{R}, L)$ при $L \rightarrow \infty$ вносит результат интегрирования в (61) по той части контура интегрирования, которая охватывает указанный разрез $(-\infty, 0]$. Обозначим через $\xi_{-}=z e^{-i \pi}$ и $\xi_{+}=z e^{i \pi}$ (где $z>0$ ) значения переменной $\xi$ соответственно на нижнем и на верхнем берегах разреза $(-\infty, 0]$. В формуле (61) учтем результат интегрирования лишь по контуру, охватывающему этот разрез. В итоге получим

$$
\widetilde{\mathcal{W}}(\mathbf{R}, L) \sim\left[\int_{0}^{\infty} d z e^{-L k_{0} z} \Delta \tilde{\varphi}(\eta)\right]^{-1} \int_{0}^{\infty} d z e^{-L k_{0} z} \Delta \tilde{\psi}(\mathbf{R}, \eta), \quad L \rightarrow \infty
$$

где

$$
\begin{aligned}
& \Delta \tilde{\varphi}(\eta) \equiv \tilde{\varphi}\left(0, \eta_{+}\right)-\tilde{\varphi}\left(0, \eta_{-}\right)= \\
&= A \Gamma(1-\mu) 2^{-\mu}\left(\eta_{+}^{2(\mu-1)}-\eta_{-}^{2(\mu-1)}+\frac{l^{2}}{4 \mu}\left(\eta_{+}^{2 \mu}-\eta_{-}^{2 \mu}\right)+O\left(\eta^{2} l^{2(2-\mu)}\right)\right), \\
& \Delta \tilde{\psi}(R, \eta) \equiv \tilde{\psi}\left(R, \eta_{+}\right)-\tilde{\psi}\left(R, \eta_{-}\right)= \\
&=\frac{A \Gamma(\nu)}{(2 \pi)^{d / 2} 2^{1-\nu}}\left(\frac{\Gamma(1-\nu)}{2^{2 \nu} \Gamma(1+\nu)}\left(\eta_{-}^{2 \nu}-\eta_{+}^{2 \nu}\right)+\frac{\varrho^{2(1-\nu)}}{4(1-\nu)}\left(\eta_{+}^{2}-\eta_{-}^{2}\right)+O\left(\varrho^{2} \eta^{2(1+\nu)}\right)\right),
\end{aligned}
$$


а переменные $\eta_{-}=\eta_{-}(z)$ и $\eta_{+}=\eta_{+}(z)$ согласно формуле (65) связаны с $z$ вблизи точки $z=0$ на нижнем и верхнем берегах разреза $(-\infty, 0]$ :

$$
\begin{aligned}
z e^{-i \pi} & =\eta_{-}^{2 \nu}+O\left(\eta_{-}^{2(3 \nu-s-1)}\right), & & \eta_{-} \rightarrow 0, \\
z e^{i \pi} & =\eta_{+}^{2 \nu}+O\left(\eta_{+}^{2(3 \nu-s-1)}\right), & & \eta_{+} \rightarrow 0 .
\end{aligned}
$$

Асимптотическая оценка интегралов в (66) при $L \rightarrow \infty$ дает

$$
\widetilde{\mathcal{W}}(\mathbf{R}, L) \sim C_{0} \frac{\left(R^{2}+l^{2}\right)^{1-\nu}}{\left(k_{0} L\right)^{(2-\mu) / \nu}}\left[\frac{e^{i \pi / \nu}-e^{-i \pi / \nu}}{e^{i \pi(\mu-1) / \nu}-e^{-i \pi(\mu-1) / \nu}}\right],
$$

где $C_{0}$ - вполне определенная положительная постоянная. Согласно условию (62) величина в квадратных скобках в правой части асимптотического выражения (68) должна быть положительной. Последнее требование соблюдается при выполнении равенства

$$
\frac{\pi}{\nu}+\frac{\pi}{\nu}(1-\mu)=2 \pi n, \quad n=1, \ldots,
$$

которое совместно с условием $\nu=\mu+s$ лишь для $n=1$. Отсюда находим

$$
\mu=\frac{2}{3}(1-s)=\frac{4-d}{3}, \quad \nu=\frac{s+2}{3}=\frac{d+2}{6} .
$$

Таким образом, искомая асимптотика плотности $\widetilde{\mathcal{W}}(\mathbf{R}, L)$ при $L \rightarrow \infty$ имеет следующий вид:

$$
\widetilde{\mathcal{W}}(\mathbf{R}, L) \sim C_{0}\left(R^{2}+l^{2}\right)^{(1-s) / 3}\left(k_{0} L\right)^{-2}
$$

Отметим, что различие в поведении функций $\widetilde{\mathcal{W}}(\mathbf{R}, L)$ в $(69)$ и асимптотики плотности в формуле $(12)$ при $L \rightarrow \infty$ уменьшается, когда размерность $d$ растет, приближаясь к $d=4$. Этот факт объясняется ослаблением эффекта от запрета на самопересечения струны с ростом размерности пространства.

Далее определим для $X$ приближение $\widetilde{X}$ с помощью равенства

$$
\tilde{\varphi}=\frac{\tilde{X}}{l \chi^{2}}
$$

аналогичного (51), и выясним, в каком смысле функцию $\widetilde{X}$ можно рассматривать в качестве решения уравнения

$$
\widetilde{X}^{-1}=1+\frac{\Psi\left(\chi^{2}, \eta^{2} ; v ; \tilde{\varphi}\right)}{l \chi^{2}} .
$$

Для этого рассмотрим асимптотическое поведение функции $\widetilde{X}^{-1}$ в точке $\eta=0$ при $p \rightarrow 0$. С одной стороны, из уравнений (59) и (70) следует, что в точке нормировки $p^{2}=\lambda$, где $\left.\widetilde{X}(p, 0)\right|_{p^{2}=\lambda}=1$, мы имеем

$$
\left.\frac{\partial \widetilde{X}(p, 0)}{\partial \ln p^{2}}\right|_{p^{2}=\lambda}=\mu+O\left(\lambda l^{2}\right)
$$


С другой стороны, если для описания асимптотики правой части уравнения (71) в точке $\eta=0$ при $p \rightarrow 0$ воспользоваться выражениями (53) и (63), то

$$
\begin{aligned}
\Psi\left(p^{2}, 0 ; v ; \tilde{\varphi}\right)= & A_{0} l^{-2 \nu} v_{0}\left(\Lambda_{s+1}\left(r_{0} p\right)-1\right)+ \\
& +A_{1} l^{-2} v_{0}^{2}\left(\bar{K}_{1}(0)-\Lambda_{s+1}^{2}\left(r_{0} p\right) \bar{K}_{1}(l p)\right)+O\left(l^{2} p^{2}\right)
\end{aligned}
$$

где при $z \rightarrow 0$

$$
\begin{aligned}
\Lambda_{s+1}(z) & =1-\frac{z^{2}}{4(s+2)}+O\left(z^{4}\right) \\
\bar{K}_{1}(z) & =1+\frac{z^{2}}{2} \ln \frac{z}{2}+(2 \gamma-1)\left(\frac{z}{2}\right)^{2}+O\left(z^{4} \ln z\right)
\end{aligned}
$$

здесь $\gamma-$ постоянная Эйлера-Маскерони. Отсюда следует, что

$$
\begin{aligned}
& \left(l p^{2}\right)^{-1} \Psi\left(p^{2}, 0 ; v ; \tilde{\varphi}\right)= \\
& \quad=-\mathcal{C} A^{3} l^{-1} v_{0}^{2} \ln \left(l^{2} p^{2}\right)+\Omega\left(A, r_{0}, l\right)+O\left(r_{0}^{2} p^{2} \ln \left(l^{2} p^{2}\right)\right), \quad p \rightarrow 0
\end{aligned}
$$

где не зависящая от $p^{2}$ величина $\Omega\left(A, r_{0}, l\right)$ связывает параметры $A, r_{0}, l$, а постоянная $\mathcal{C}$ задается формулой

$$
\mathcal{C}=\frac{\Gamma^{3}(\nu)}{4(2 \pi)^{d} \Gamma(3 \nu)} .
$$

Тогда из уравнения (71) и выражения (73) следует, что

$$
\left.\frac{\partial \tilde{X}(p, 0)}{\partial \ln p^{2}}\right|_{p^{2}=\lambda}=\mathcal{C} A^{3} l^{-1} v_{0}^{2}+O\left(\lambda r_{0}^{2}\right)
$$

Сравнивая правые части равенств (72) и (74), мы получаем, что $A=C_{A}\left(l v_{0}^{-2}\right)^{1 / 3}$, где $C_{A}$ - вполне определенная положительная постоянная.

Таким образом, значения всех параметров задачи определены, и мы можем выписать искомую асимптотику плотности вероятности $\widetilde{\mathcal{W}}(\mathbf{R}, L)$ в пространстве $\mathbb{R}^{d}$ при $2 \leqslant d<4$, когда $R / l \rightarrow \infty$ и $L / l \rightarrow \infty$, но их отношение $R / L$ фиксировано и мало. Используя формулы (59)-(61) и (64), находим главный член асимптотического разложения функции $\widetilde{\mathcal{W}}(\mathbf{R}, L)$ :

$$
\widetilde{\mathcal{W}}(\mathbf{R}, L) \sim C_{d}\langle\varrho\rangle^{-d} \mathcal{Z}^{(4-d) D / 6} e^{-c_{d} \mathcal{Z}^{D}}
$$

где

$$
\langle\varrho\rangle=\left(k_{0} L\right)^{3 /(d+2)}, \quad \mathcal{Z} \equiv \frac{\varrho}{\langle\varrho\rangle}=\frac{\sqrt{R^{2}+l^{2}}}{\left(k_{0} L\right)^{3 /(d+2)}}, \quad D=\frac{d+2}{d-1},
$$

a $c_{d}$ и $C_{d}$ - вполне определенные постоянные. Случай $d=4$ является особым, и его следует рассматривать отдельно. Тем не менее полученный результат позволяет предположить, что в случае $d>4$ асимптотика плотности вероятности при рассмотренных выше условиях имеет форму нормального распределения. Это означает, что в этом случае условие отсутствия самопересечений оказывает несущественное влияние на распределение расстояния между концами струны [6], [7]. 


\section{6. КАНОНИЧЕСКИЙ АНСАМБЛЬ ОТКРЫТЫХ СТРУН}

Рассмотрим теперь модель ансамбля из $M$ струн, средняя длина которых по всем струнам равна заданной величине $\bar{L}$, и найдем наиболее вероятное распределение струн по их длинам в предельном случае $M \rightarrow \infty$. Для вывода канонического ансамбля мы воспользуемся известным методом Дарвина-Фаулера [8]. Допустим, что длины струны в ансамбле могут принимать любое из значений $L_{k}=k L$; здесь $k=0,1,2, \ldots$, a $L$ - масштабная длина струны. Если в ансамбле $m_{k}$ струн имеют длину $L_{k}$, то набор целых чисел $\left\{m_{k}\right\}, k=0,1,2, \ldots$, описывает любое распределение их длин. При этом должны выполняться следующие условия:

$$
\sum_{k=0}^{\infty} m_{k} \equiv \Xi=M, \quad \sum_{k=0}^{\infty} k m_{k} \equiv \bar{\Xi}=M \bar{N},
$$

где $\bar{N} \equiv \bar{L} / L \gg 1$ и $M$ суть целые числа. Пусть $W\left\{m_{k}\right\}$ обозначает число различных способов распределения длин по струнам при соблюдении условий (75). Очевидно, что

$$
W\left\{m_{k}\right\}=\frac{M !}{m_{0} ! m_{1} ! m_{2} ! \ldots} .
$$

Согласно аксиоме равных априорных вероятностей в данном случае можно утверждать, что все распределения длины между струнами имеют одинаковую вероятность, если выполнены условия (75). Тогда средние значения величин $m_{k}$ по всем распределениям длин определяются как

$$
\left\langle m_{k}\right\rangle \equiv \frac{\sum_{\left\{m_{j}\right\}} m_{k} W\left\{m_{j}\right\}}{\sum_{\left\{m_{j}\right\}} W\left\{m_{j}\right\}},
$$

где суммирование выполняется по всем наборам $\left\{m_{k}\right\}$, удовлетворяющим равенствам (75).

Пусть $\left\{\bar{m}_{k}\right\}$ есть множество, соответствующее максимальному значению величины $W\left\{m_{k}\right\}$. Если $\left\langle m_{k}^{2}\right\rangle \rightarrow\left\langle m_{k}\right\rangle^{2}$ при $M \rightarrow \infty$, то в этом предельном случае $\left\langle m_{k}\right\rangle \rightarrow\left\langle\bar{m}_{k}\right\rangle$. Чтобы вычислить величину $\left\langle m_{k}\right\rangle$ с помощью формулы (76), удобно воспользоваться более общим выражением

$$
W^{\prime}\left\{m_{k}\right\}=M ! \frac{\lambda_{0}^{m_{0}} \lambda_{1}^{m_{1}} \lambda_{2}^{m_{2}} \cdots}{m_{0} ! m_{1} ! m_{2} ! \ldots},
$$

а числа $\lambda_{k}, k=0,1,2, \ldots$, в конце вычислений положить равными единице. Принимая во внимание условие (75) при вычислении суммы

$$
S(M, \bar{N}) \equiv \sum_{\left\{m_{k}\right\}} W^{\prime}\left\{m_{k}\right\},
$$

воспользуемся формулами Коши

$$
\begin{gathered}
\frac{1}{2 \pi i} \oint_{\Gamma} d \zeta \zeta^{\Xi-M-1}=\left\{\begin{array}{lll}
1, & \text { если } & \Xi=M, \\
0, & \text { если } & \Xi \neq M,
\end{array}\right. \\
\frac{1}{2 \pi i} \oint_{\gamma} d z z^{\bar{\Xi}-M \bar{N}-1}=\left\{\begin{array}{lll}
1, & \text { если } & \bar{\Xi}=M \bar{N}, \\
0, & \text { если } & \bar{\Xi} \neq M \bar{N},
\end{array}\right.
\end{gathered}
$$


где замкнутые контуры Г и $\gamma$ охватывают начала координат в плоскостях комплексных переменных $\zeta$ и $z$ соответственно. Отсюда следует, что

$$
S(M, \bar{N})=\frac{M !}{(2 \pi i)^{2}} \oint_{\gamma} d z z^{-M \bar{N}-1} \oint_{\Gamma} d \zeta \zeta^{-M-1} e^{\zeta h(z)}=\frac{1}{2 \pi i} \oint_{\gamma} d z z^{-M \bar{N}-1} h^{M}(z),
$$

где

$$
h(z)=\sum_{k=0}^{\infty} \lambda_{k} z^{k}
$$

Далее, используя метод перевала для асимптотической оценки последнего интеграла в (77) при $M \rightarrow \infty$, мы получим

$$
S(M, \bar{N}) \approx \frac{1}{\sqrt{2 \pi M H^{\prime \prime}\left(z_{0}\right)}} e^{M H\left(z_{0}\right)}, \quad M \rightarrow \infty,
$$

где

$$
\begin{aligned}
H\left(z_{0}\right) & =\ln h\left(z_{0}\right)-\left(\bar{N}+M^{-1}\right) \ln z_{0}, \\
H^{\prime \prime}\left(z_{0}\right) & =\frac{h^{\prime \prime}\left(z_{0}\right)}{h\left(z_{0}\right)}-\frac{\left(\bar{N}+M^{-1}\right)\left(\bar{N}+M^{-1}-1\right)}{z_{0}^{2}},
\end{aligned}
$$

а $z_{0}$ - положительный корень уравнения $H^{\prime}\left(z_{0}\right)=0$. Отсюда следует, что

$$
\frac{1}{M} \ln S(M, \bar{N}) \rightarrow H\left(z_{0}\right), \quad M \rightarrow \infty
$$

и тогда, принимая во внимание это соотношение, а также равенство

$$
\left\langle m_{k}\right\rangle=\lambda_{k} \frac{\partial \ln S(M, \bar{N})}{\partial \lambda_{k}}
$$

мы получим

$$
\lim _{M \rightarrow \infty} \frac{\left\langle m_{k}\right\rangle}{M} \equiv \mathrm{P}(k)=\frac{e^{-\xi k}}{\sum_{n=0}^{\infty} e^{-\xi n}},
$$

где $\xi=-\ln z_{0}$. Пользуясь этой формулой, можно показать, что $\left\langle m_{k}\right\rangle=\left\langle\bar{m}_{k}\right\rangle$ при $M \rightarrow \infty$. Параметр $\xi$ здесь определяется уравнением

$$
\bar{N}=\mathrm{E} k \equiv \sum_{k=0}^{\infty} k \mathrm{P}(k)
$$

из которого следует, что $z_{0}=\bar{N} /(\bar{N}+1)$. Учитывая уравнение $(79)$, усредним равенство (43) по распределению (78):

$$
\mathrm{E} W(\mathbf{R}, L) \equiv \sum_{k=0}^{\infty} W\left(\mathbf{R}, L_{k}\right) \mathrm{P}(k)=\bar{L}^{-1} \psi\left(\mathbf{R}, \bar{L}^{-1}\right)
$$

Далее мы определим плотность распределения

$$
\langle W(\mathbf{R}, L)\rangle \equiv\left(\int_{\mathbb{R}^{d}} d \mathbf{R} \mathrm{E} W(\mathbf{R}, L)\right)^{-1} \mathrm{E} W(\mathbf{R}, L) .
$$


Отсюда с учетом формул (44), (80) вытекает,что

$$
\langle W(\mathbf{R}, L)\rangle=\frac{\psi\left(\mathbf{R}, \bar{L}^{-1}\right)}{\varphi\left(0, \bar{L}^{-1}\right)} .
$$

Следуя изложенному выше подходу, воспользуемся в этом соотношении приближением (59) и получим соответствующее приближение для $\langle W(\mathbf{R}, L)\rangle$ :

$$
\langle\widetilde{\mathcal{W}}(\mathbf{R}, L)\rangle=\frac{\tilde{\psi}(R, \eta)}{\tilde{\varphi}(0, \eta)},
$$

где связь между $\eta$ и $\bar{L}$ задается как

$$
\bar{L}^{-1}=k_{0} \eta^{2 \nu}+O\left(l \eta^{2}\right), \quad \eta \rightarrow 0
$$

в соответствии с равенством (64). В результате имеем

$$
\langle\widetilde{\mathcal{W}}(\mathbf{R}, L)\rangle=\widetilde{C}_{d} \varrho^{-\alpha} \tilde{L}^{-\beta} K_{\nu}(\varrho / \tilde{L}),
$$

где $\widetilde{C}_{d}$ - определенная положительная постоянная, $\varrho^{2}=l^{2}+R^{2}$,

$$
\alpha=\frac{d-1}{3}, \quad \beta=\frac{2 d+1}{3}, \quad \nu=\frac{d+2}{6}, \quad \tilde{L}=\left(k_{0} \bar{L}\right)^{1 / 2 \nu} .
$$

Таким образом, формула (81) описывает распределение канонического ансамбля открытых струн, избегающих самопересечения в $\mathbb{R}^{d}$ при $2 \leqslant d<4$.

\section{Список литературы}

[1] В. И. Алхимов, Фундамент. и прикл. матем., 17:5 (2012), 3-20.

[2] Е. Титчмарш, Теория функиий, Наука, М., 1980.

[3] Н. Н. Боголюбов, Д. В. Ширков, Введение в теорию квантованных полей, Наука, М., 1976.

[4] Л. В. Овсянников, Групповой анализ дифференциальных уравнений, Наука, М., 1978.

[5] M. Gell-Mann, F. E. Low, Phys. Rev., 95:5 (1954), 1300-1312.

[6] D. Bridges, T. Spencer, Commun. Math. Phys., 97:1-2 (1985), 125-148.

[7] T. Hara, G. Slade, Rev. Math. Phys., 4:2 (1992), 235-327.

[8] К. Хуанг, Статистическая механика, Мир, М., 1966.

Поступила в редакцию 16.12.2013, после доработки 18.03.2014 